\title{
Functional Outcome of Distraction Osteogenesis Using Linear Rail System (LRS) in Adults with Isolated Femoral Bone Gap
}

\section{Alabi IA*, Okoh N, MN Salihu, Mustapha IU, Musa NT, Muhammad AM, Jimoh LA, Aroju SA and Ibrahim Y}

Department of Clinical Services, National Orthopaedic Hospital, Nigeria

*Corresponding author: Dr Alabi Ibrahim Abolaji, Department of Clinical Services, National Orthopaedic Hospital, Nigeria, Email: ibrahimic75@yahoo.com

\section{Research Article \\ Volume 5 Issue 1}

Received Date: March 20, 2021

Published Date: May 04, 2021

DOI: $10.23880 /$ jobd-16000208

\section{Abstract}

Background: The management of bone gap in the femoral shaft has remained difficult and challenging to the orthopaedic and trauma Surgeons. Distraction osteogenesis using the linear rail system (LRS) may be a useful tool in bridging such defects in our environment.

Objective: To study the radiologic bone union, functional outcome and complications of distraction osteogenesis in the management of femoral bone gap using linear rail system (LRS).

Methodology: A hospital based prospective interventional study conducted at National Orthopaedic Hospital, Dala and Albarka clinic, Dandishe, Kano, between March 2013 and March 2018. Sixty-eight cases were recruited. Every patient had LRS applied after adequate debridement when needed. Primary or interval corticotomy was done depending on the extent of soft tissue dissection in relation to the corticotomy site. Follow up was done until the regenerate had corticalized and LRS removed. Complications were noted and recorded. Radiologic union and functional outcome were assessed using the RUST and ASAMI outcome scores respectively. Data was analyzed using SPSS version 20.

Results: Sixty-eight cases were recruited with M: F ratio of 16: 1 . The mean age was $36.7+/-10.5 y e a r s$. The age range between 36-45years was more commonly treated representing 34(50.0\%). Primary corticotomy was done in 52 (76.5\%) while interval corticotomy was done in $16(23.5 \%)$. The mean regenerate length achieved was $9.6+/-2.7 \mathrm{~cm}$. The mean duration of treatment was $20.3+/-6$ months. Radiologic union was achieved in 66 (97.1\%) patients. Sixty-four (94.1\%) had excellent or good ASAMI functional outcome scores. Sixty-six (97.1\%) were either very satisfied or satisfied with their treatment while $2(2.9 \%)$ were indifferent. The common complications were intermittent pin tract infections recorded in $28(41.2 \%)$, knee stiffness in 18 (26.5), proximal or distal varus deformity in 10 (14.7\%) and non-union in $2(2.9 \%)$ of cases.

Conclusion: Distraction osteogenesis using LRS can achieve bone union and excellent outcome in the management of bone gap in the femur. However, intermittent pin tract infection, knee stiffness and varus deformities post significant challenges.

Keywords: Functional Outcome; Femoral Bone Gap; Linear Rail System 


\section{Journal of Orthopedics \& Bone Disorders}

Level of evidence: level II (prospective cohort study)

Abbreviations: LRS: Linear Rail System; LLD: Limb Length Discrepancy; GA: Gustilo and Anderson type; ATLS: Advanced Trauma Life Support; POP: Plaster of Paris.

\section{Introduction}

The term distraction osteogenesis describes the induction of new bone formation between the cut surfaces of two bone fragments that are gradually pulled apart. This intrinsic capacity of bone to regenerate is harnessed to lengthen bone or bridge large segments of bone loss [1].

The management of long bone fractures with significant bone defects and limb length discrepancy (LLD) by distraction osteogenesis can pose a major challenge to the orthopedic surgeon due to associated high complication rates, which can be classified as problems, obstacles or complications [2]. Some problems are post-operative events that may require no surgical intervention to resolve them, such as superficial pin tract infection, while obstacles are postoperative events that are resolved only after operative intervention, such as premature consolidation [2]. True complications are those adverse effects occurring intra-operatively or those which fail to resolve despite operative intervention['[2].

The specific method of treatment of these fractures is a matter of debate, with the treatment options ranging from external fixators, ring fixators, nailing, plating, free or vascularized bone grafting along with allografts or bone substitutes, all having their own set of complications [35]. Traditional treatment recommendations for bridging segmental bone loss by autogenous bone grafting is associated with long remodeling time and high fracture rates, hence weight bearing during the course of treatment is not recommended [6]. Autogenous bone grafting is also associated with donor site morbidity [7].

The linear rail system (LRS) consists of an assembly of clamps (usually two or three), which can slide on a rigid rail and can be connected by compression-distraction units to achieve bone transport. The linear rail system (LRS) uses the principle of distraction osteogenesis for bridging bone gaps. It is relatively easy to learn and can be rapidly applied [8]. It also allows functional weight bearing with dynamic compression [9].

In this prospective study, we present our experience of using LRS in the treatment of femoral bone gap as a definite mode of treatment to achieve union, as well as limb lengthening simultaneously in a Nigerian population with particular reference to functional outcome, bone union and identifiable complications.

\section{Methodology}

A hospital based prospective interventional nonrandomized study conducted at National Orthopaedic Hospital, Dala, and Albarka clinic, Dandishe, Kano. The two hospitals receive patients from all over Nigeria and some neighboring countries like Chad, Niger and Cameroon. The study was conducted between March 2013 and March 2018. Sixty-eight cases of fractures of femoral diaphysis with bone loss were recruited as they presented and treated using LRS to achieve bone transport, union and lengthening when necessary. Every patient had LRS applied after adequate debridement when required. The inclusion criteria were patients between 18 and 65 years, Gustilo and Anderson type III A and III B with bone loss of $3 \mathrm{~cm}$ or more. Infected nonunion and failed osteosynthesis with shortening $>/=3 \mathrm{~cm}$ were also included. Gustilo and Anderson type (GA) IIIC, multiply injured patients, patients with loss of cognitive function and those who refused to give consent were excluded from the study. Ethical clearance was obtained from the institution research and ethics committee.

In patients presenting with post-traumatic bone loss in the acute setting, primary survey was done to rule out associated injuries and prevent life threatening complications. Resuscitation was done using Advanced Trauma Life Support (ATLS) protocol, thorough irrigation of the wound, dressing with sterile materials and primary splintage with Plaster of Paris (POP) backslab. Third generation intravenous cephalosporin was given. Preoperative workup involved taking orthogonal plain radiographs of the affected thigh with hip and knee joints clearly outlined.

All patients had adequate surgical debridement when indicated. Patients with failed osteosynthesis had removal of implant in addition to surgical debridement. Linear rail system (LRS) was applied in all cases. Schantz pins of LRS were inserted following longitudinal stab incision, separation of the soft tissue down to the bone by blunt dissection, and introduction of a drill guide. Schantz pin (6.0 $\mathrm{mm}$ in diameter) was inserted by sequentially predrilling with an appropriate-size 'drill bit' $(4.5 \mathrm{~mm}$ in diameter), followed by manual insertion of the Schantz screw using T-handle over. The proximal and then the distal most pins were inserted first followed by sequential arrangement of the other pins assembled along the anterolateral plain of the thigh. Leg length was maintained where achievable, axial and rotational alignment of proximal and distal bone fragments was ensured.

Primary or interval corticotomy was done depending on the extent of soft tissue dissection in relation to the corticotomy site. This was achieved via low energy osteotomy. Postoperatively systemic antibiotic was continued for 2 days, 


\section{Journal of Orthopedics \& Bone Disorders}

then oral antibiotics for 2 weeks, and dressing done regularly. In patients with soft tissue defect, they had primary wound cover if wound was clean and no sign of infection otherwise delayed wound closure was done as wound condition improved. If corticotomy is done, distraction is commenced until wound cover is achieved. Wound cover was achieved if required, using split skin grafting or flaps as required.

Distraction was started after 7-10days of the corticotomy at the rate of $1 \mathrm{~mm} /$ day using 6 hourly rhythms. Physiotherapy was commenced to maintain/improve hip and knee range of motion (ROM) after the application of LRS, depending on the patient's pain tolerance. A check X-ray is done after a week of distraction and patients were discharged to continue subsequent distraction on outpatient basis. Bone transport was continued till the fracture ends were docked. Following docking, the distraction was stopped and patients were commenced on weight bearing as tolerated. Patients with clinically significant limb length discrepancies had lengthening following transport, each patient was followed up until regenerate had corticalized and LRS removed. Corticalized regenerate was defined by presence of minimum of 3 cortices in orthogonal views using RUST score [10-12].

Complications during treatment were observed and documented during the follow-up period. Patients' were taught daily pin tract care. Functional outcome was assessed using Association for the study and application of the method of Ilizarov (ASAMI) score. Data was collated and analyzed using SPSS version 20.

\section{Results}

Sixty-eight cases were recruited with M: F ratio of 16: 1 . The mean age was $36.7+/-10.5$ years. The age range between 36-45years was more commonly treated representing Table 1 below shows patients' age distribution. Figure 1 below shows the patients' mode of presentation. Posttraumatic segmental bone loss accounted for 28 (58.3\%) while infected implant accounted for $15(31.3 \%)$ of the cases. Primary corticotomy was done in $52(76.5 \%)$ while interval corticotomy was done in $16(23.5 \%)$ (Figure 2).

\begin{tabular}{|c|c|c|}
\hline Age Distribution (Years) & Frequency & Percent \\
\hline $18-25$ & 6 & 14.6 \\
\hline $26-35$ & 18 & 29.2 \\
\hline $36-45$ & 34 & 45.8 \\
\hline $46-55$ & 3 & 2.1 \\
\hline $56-65$ & 7 & 8.3 \\
\hline Total & 68 & 100 \\
\hline
\end{tabular}

Table 1: Age distribution.

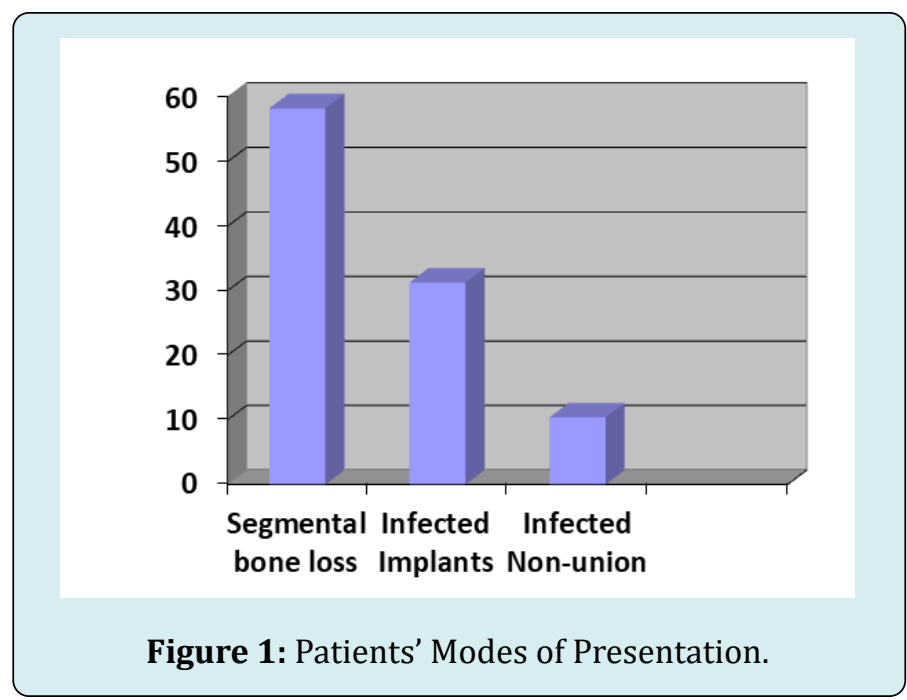

Primary corticotomy was done in 36 (75\%) while interval corticotomy was done in 12 (25\%), see figure 2 below.

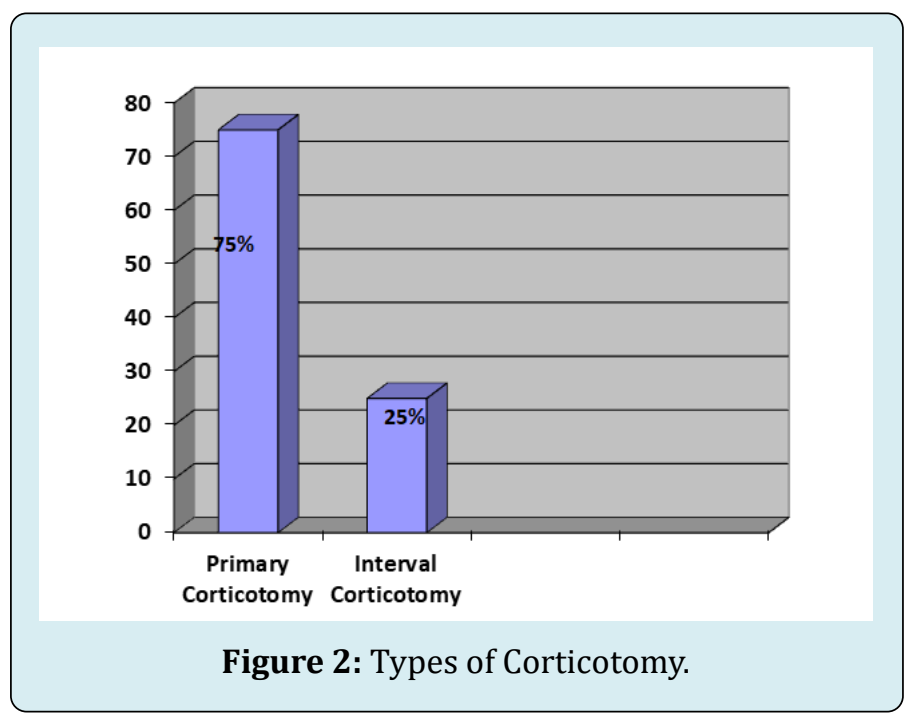

The mean regenerate length achieved was $9.6+/-2.7 \mathrm{~cm}$ (Table 2). The mean duration of treatment was $20.3+/-6$ months (Table 3). Radiographs and clinical photographs of a typical case are as shown in fig $3 a-e$ and $4 a-d$ respectively

\begin{tabular}{|c|c|c|}
\hline Regenerate Length (CM) & Frequency & Percent \\
\hline $4-9$ & 32 & 47.1 \\
\hline $10-14$ & 34 & 50 \\
\hline $15-20$ & 2 & 2.9 \\
\hline Total & 68 & 100 \\
\hline
\end{tabular}

Table 2: Regenerate length. 
Journal of Orthopedics \& Bone Disorders

\begin{tabular}{|c|c|c|}
\hline Duration of Treatment (Months) & Frequency & Percent \\
\hline $07-12$ & 3 & 4.4 \\
\hline $13-18$ & 36 & 52.9 \\
\hline $19-24$ & 21 & 30.9 \\
\hline $25-30$ & 3 & 4.4 \\
\hline $31-36$ & 5 & 7.4 \\
\hline Total & 68 & 100 \\
\hline
\end{tabular}

Table 3: Duration of Treatment.
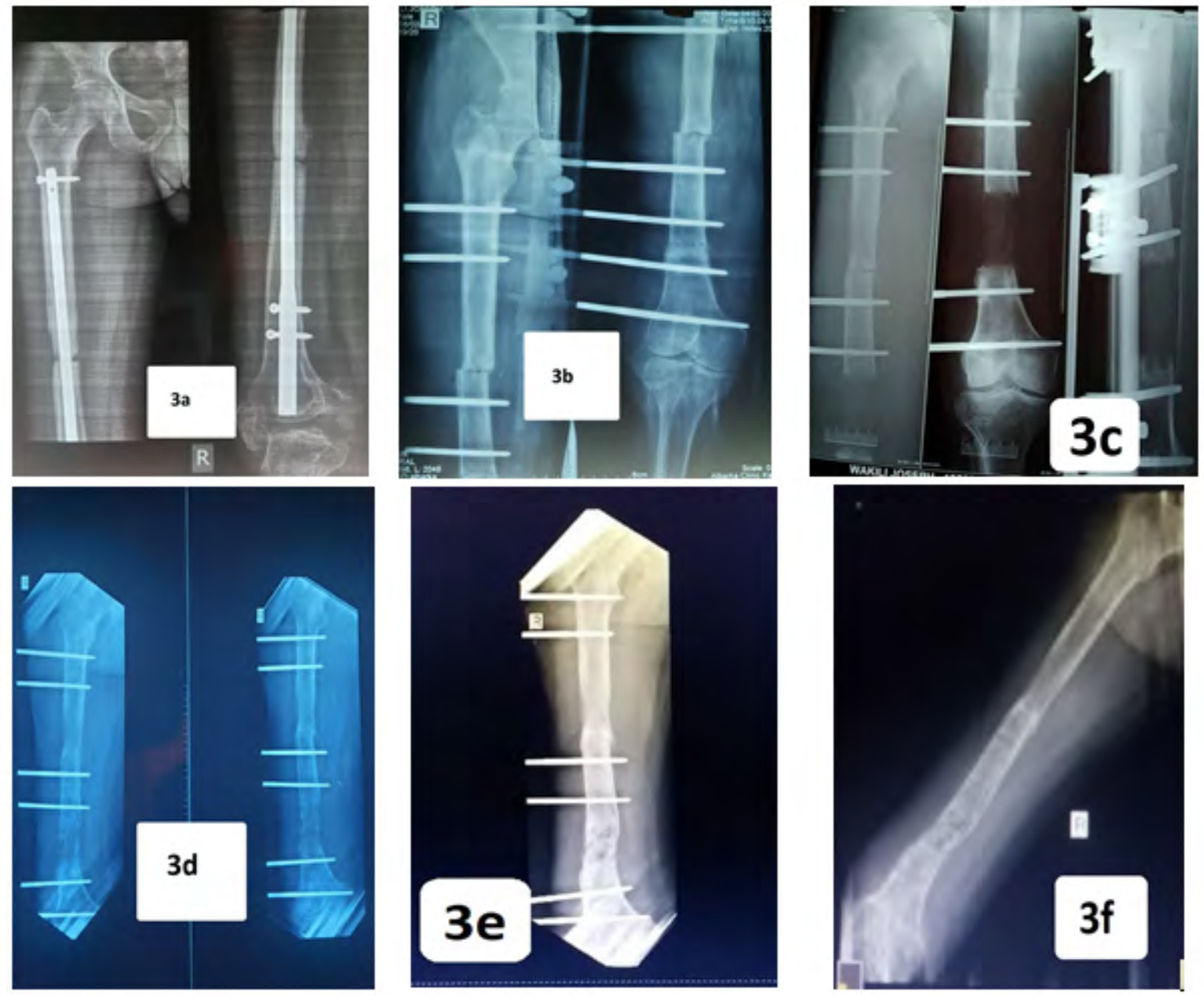

Figure 3: Radiographs (a) Preoperative period (infected left femoral IM Nail), (b) Postoperative radiographs with LRS and corticotomy done, (c) Post-distraction (d) at early consolidation (e) Post consolidation of the regenerate, (f) Post removal of LRS. 

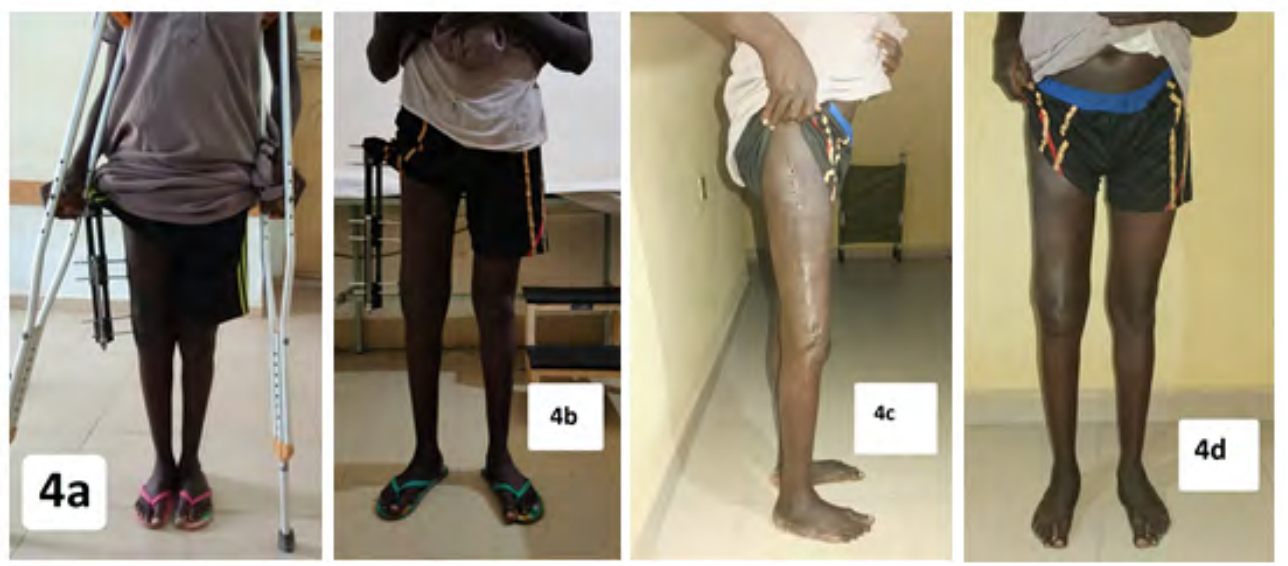

Figure 4: (a) The ambulant patient with LRS on his right femur. (b) Walking unaided before removal of the LRS (c, d) After removal of LRS.

\begin{tabular}{|c|c|c|}
\hline ASAMI Functional Outcome Score & Frequency & Percent \\
\hline Excellent & 51 & 75 \\
\hline Good & 13 & 19.1 \\
\hline Fair & 4 & 5.9 \\
\hline Total & 68 & 100 \\
\hline
\end{tabular}

varus deformity in $10(14.7 \%)$ and non-union in $2(2.9 \%)$ of cases, See Figure 6. Figure 7 shows a case of proximal varus angulation corrected using a locked intramedullary nail.

Table 4: ASAMI Functional Outcome Score.

Radiologic union was achieved in 66 (97.1\%) patients. Sixty-four (94.1\%) had excellent or good ASAMI functional outcome scores (Table 4). Sixty-six (97.1\%) were either very satisfied or satisfied with their treatment while 2 (2.9\%) were indifferent (Figure 5).

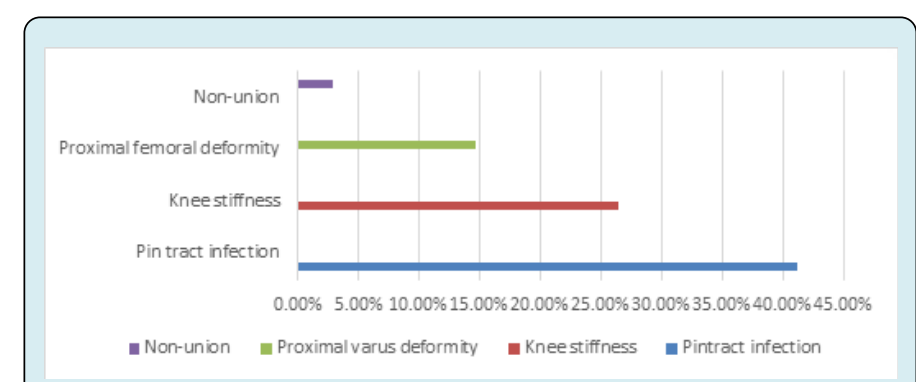

Figure 6: Showing various complications.
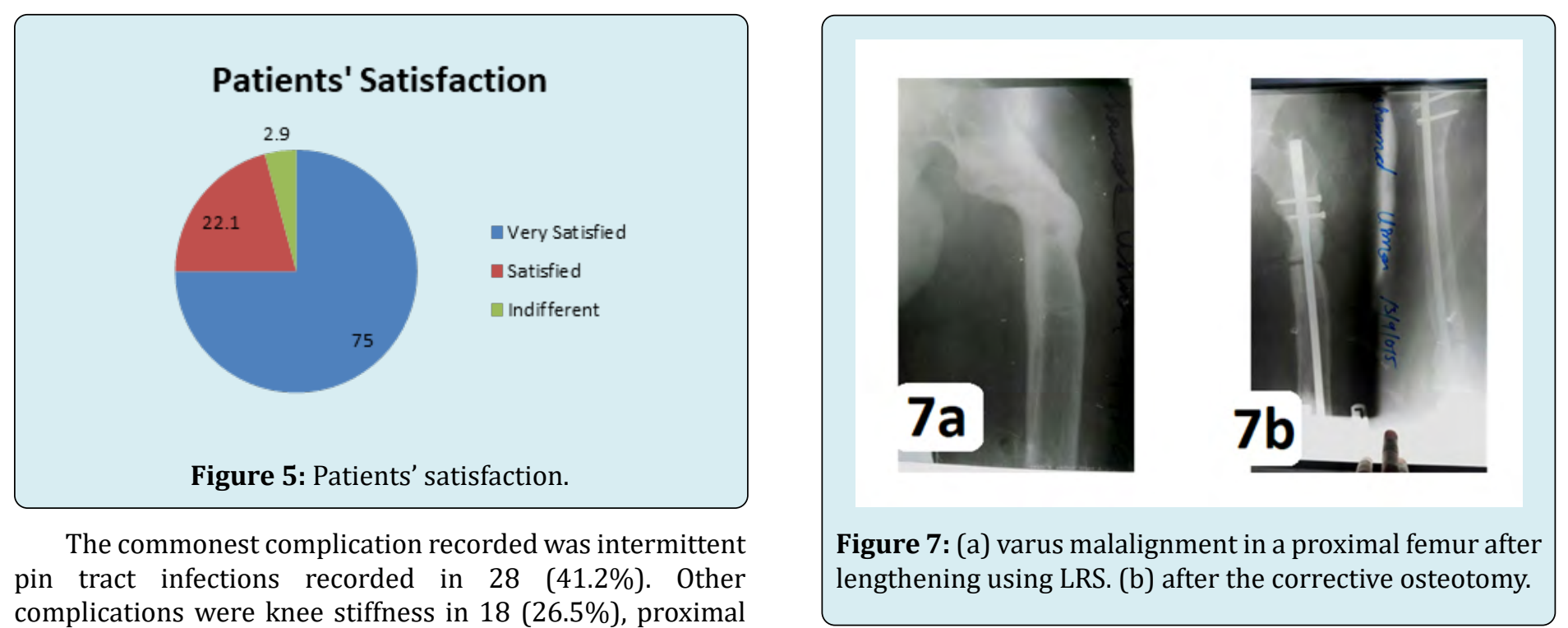
pin tract infections recorded in $28(41.2 \%)$. Other
complications were knee stiffness in $18(26.5 \%)$, proximal 


\section{Journal of Orthopedics \& Bone Disorders}

\section{Discussion}

Distraction osteogenesis using linear rail system (LRS) in the presence of bone loss or LLD is relatively easy to learn and can be rapidly applied]"[8]. LRS is designed primarily for limb lengthening and in nonunion and deformity correction, which includes different types of clamps (usually two or three) that can slide on a rigid rail and are connected with compression and distraction units [13]. LRS uses osseous callus distraction for bone lengthening in a variety of procedures such as bone transport, simultaneous compression and distraction at different sites, monofocal lengthening, bifocal lengthening and correction of deformities with shortening[ [10].

We evaluated the functional outcome of LRS in 68 cases of femoral bone gap resulting from trauma or following debridement in cases of infected nonunion and failed osteosynthesis with shortening. With mean age of $36.7+/-10.5 y e a r s$ with an average treatment duration of 20.3 $+/-6$ months and mean regenerate length of $9.6+/-2.7 \mathrm{~cm}$, LRS was used as a definitive method of treatment for achieving union as well as for lengthening done simultaneously where required. The above biodata and male preponderance were similar to the findings of Donwa, et al. [14].

Corticotomy in our study was achieved by single incision low energy osteotomy using a corticotome with preservation of periosteum, it has been shown that preserving periosteum, rather than medullary circulation and low energy osteotomy are the two most important determinants for good quality regenerate [15]. This study showed excellent to good functional outcome result in $93.8 \%$ of cases which was comparable to results obtained by Alabi, et al. [16] and Anand, et al. [13]. Who obtained results of $94.6 \%$ and $84 \%$ respectively. This study is also comparable to results from other series in which bone loss was treated by other methods like McKee, et al. [17], Donnan, et al. [18], Robert, et al. [19], and Mekhail, et al. [20] , all of which showed excellent to good results in $80-90 \%$ of cases.

In our study the mean bone regenerate length was 9.6 $\mathrm{cm}$ which was similar to results from Alabi, et al!. [15] but better than other series like Robert, et al. $(6 \mathrm{~cm})$ [18], Anand, et al. (5.5cm) [13], Mekhail, et al. (5.7 cm) [19], Donnan, et al. $(6.4 \mathrm{~cm})$ ['[17] and Bumbasirevic, et al. $(6.9 \mathrm{~cm})$ [21]. The most common complication, in concordance with other series $[14,17-20,22]$ was pin tract infection which was seen in 28 $(41.2 \%)$ of our patients. This may probably be as a result of the longer mean duration of treatment as well as inclusion of infected nonunion in the study population in our series.

Ten patients (14.7\%) had some form of varus malalignment in this series, however only one of these patients had significant malalignment which required corrective osteotomy with internal fixation using locked intramedullary nail, one year after removal of LRS. Eighteen patients $(26.5 \%)$ had various degrees of knee stiffness, two of which required quadriceplasty. Others were amenable to physiotherapy. These may be due to pains from pin tract infection limiting full range of motions. Two of the patients could not achieve bone union at the primary fracture sites necessitating open reduction and internal fixation with locked intramedullary nails. They achieved union and equal limbs subsequently. Non-union and late bowing were also reported by Dror paley [2].

Sixty-six $97.1 \%$ of the patients in this series were satisfied with their treatment and compliance was also satisfactory as all patients completed the course of treatment and follow-up, this is in contradiction to ring fixators like Illizarov whose application has a steep learning curve with a high rate of patient dissatisfaction [2]. This study does not however claim superiority of LRS over ring fixators, neither does it aim to replace it, but our study does show the effectiveness of LRS in the management of post traumatic femoral bone gap as well as infected femoral nonunion and failed osteosynthesis even when the bone loss is greater than $6 \mathrm{~cm}$. This deviates from the recommendation of Rohilla R, et al. [23].

\section{Conclusion}

Distraction osteogenesis using LRS can achieve bone union and excellent outcome in the management of bone gap in the femur. However, pin tract infection, knee stiffness and varus deformity (in distractions in the proximal 3rd of the femur) post significant challenges in our environment.

\section{References}

1. Solomon L, Warwick D, Nayagam S (2010) $9^{\text {th }}$ (Edn.), Appley's system of Orthopaedics and fractures. Hodder Arnold Hachette Company UK, London pp: 320.

2. Paley D (1990) Problems, obstacles, and complications of limb lengthening by the Ilizarov technique. Clin Orthop Relat Res 250: 81-104.

3. Watson JT, Anders M, Moed BR (1995) Management strategies for bone loss in tibial shaft fractures. Clin Orthop Relat Res 315: 138-152.

4. Maurer RC, Dillin L (1987) Multistaged surgical management of posttraumatic segmental tibial bone loss. Clin Orthop Relat Res 216: 162-170.

5. Keating JF, Simpson AH, Robinson CM (2005) The management of fractures with bone loss. J Bone Joint Surg Br 87(2): 142-150.

6. William FE, John LE, Hans B (1980) Autogenous cortical 


\section{Journal of Orthopedics \& Bone Disorders}

bone grafts in reconstruction of segmental defects. J Bone Joint Surg Am 62(7): 1039-1058.

7. Younger EM, Chapman MW (1989) Morbidity at bone graft donor site. J Orthop Trauma 3(3):192- 195.

8. Harshwal RK, Sankhala SS, Jalan D (2014) Management of non-union of lower extremity long bone using monolateral external fixator-report of 37 cases. Injury 45(3): 560-567.

9. Debastiani G, Aldegheri R, Brivio LR (1984) The treatment of fractures with dynamic external fixator. J Bone Joint Surg Br 66(4): 538-545.

10. Whelan DB, Bhandari M, Stephen D, Hans K, McKee MD, et al. (2010) Development of the radiographic union score for tibial fractures for the assessment of tibial fracture healing after intramedullary fixation. J Trauma 68(3): 629-632.

11. Kooistra BW, Dijkman BG, Busse JW, Sheila S, Emil HS, et al. (2010) The radiographic union scale in tibial fractures: reliability and validity. J Orthop Trauma 24(1): 81-86.

12. Ekansh D, Narendra SK, Dharmendra K, Ajai S, Vineet S (2019) Rust score-An adequate rehabilitation guide for diaphyseal femur fractures managed by TENS. J Clin Orthop Trauma 10(5): 922-927.

13. Anand A, Ankit V, Mukul A, Saurabh J, Arunangshu M (2015) Outcome of limb reconstruction system in open tibial diaphyseal fractures. Indian J Orthop 49(4): 429435.

14. Donwa Jo, Mue DD, Isa NA, Salihu MN, Yongu WT (2017) Management of complex long bone Non-unions using Linear rail system. DJ0 1(1): 1-9.

15. Eralp L, Kocaoglu M, Ozkan K, Turker M (2004) A comparison of two osteotomy techniques of tibial lengthening. Arch orthop trauma surg 124(5): 298-300.
16. Alabi AI, Mustapha I, Salihu MN, Okoh N (2017) Outcome of distraction osteogenesis using linear rail system in adults with isolated tibia bone gap at Albarka clinic Kano. Dala Journal of Orthopaedics 1(1): 11-17.

17. McKee MD, Yoo DJ, Zdero R, Dupere M, Wild L, et al. (2008) Combined single-stage osseous and soft tissue reconstruction of the tibia with the Ilizarov method and tissue transfer. J Orthop Trauma 22(3): 183-189.

18. Donnan LT, Saleh M, Rigby AS, McAndrew A (2002) Radiographic assessment of bone formation in tibia during distraction osteogenesis. J Pediatr Orthop 22(5): 645-651.

19. Robert RS, Weitzman AM, Tracey WJ, Freudigman P, Katz HV, et al. (2006) Simultaneous treatment of tibial bone and soft-tissue defects with the Ilizarov method. J Orthop Trauma 20(3): 197-205.

20. Mekhail AO, Abraham E, Gruber B, Gonzalez M (2004) Bone transport in the management of posttraumatic bone defects in the lower extremity. J Trauma 56(2): 368-378.

21. Bumbasirevic M, Tomic S, Lesic A, Milosevic I, Atkinson HD (2010) War-related infected tibial nonunion with bone and soft-tissue loss treated with bone transport using the Ilizarov method. Arch Orthop Trauma Surg 130(6): 739-749.

22. Wani N, Baba A, Kangoo K, Mir M (2011) Role of early Ilizarov ring fixator in the definitive management of type II, IIIA and IIIB open tibial shaft fractures. Int Orthop 35(6): 915-923.

23. Rohilla R, Wadhwani J, Devgan A, Singh R, Khanna M (2016) Prospective randomized comparison of ring versus rail fixator in infected gap nonunion of tibia treated with distraction osteogenesis; Bone Joint J 98B(10): 1399-1405. 